\title{
Oncologist and Patient Preferences for Novel Agents in First-Line Treatment for Chronic Lymphocytic Leukemia: Commonalities and Disconnects
}

This article was published in the following Dove Press journal: Patient Preference and Adherence

\author{
Hannah Le \\ Kellie Ryan' \\ Svea K Wahlstrom ${ }^{2}$ \\ Martine C Maculaitis ${ }^{3}$ \\ Oliver Will ${ }^{4}$ \\ Emily Mulvihill ${ }^{5}$ \\ Thomas W LeBlanc ${ }^{6}$ \\ 'US Medical Affairs, AstraZeneca, \\ Gaithersburg, MD, USA; ' ${ }^{2}$ US Patient \\ Safety, AstraZeneca, Wilmington, DE, \\ USA; ${ }^{3}$ Kantar, Health Division, New York, \\ NY, USA; ${ }^{4}$ Kantar, Health Division, \\ Horsham, PA, USA; ${ }^{5}$ Kantar, Health \\ Division, St. Louis, MO, USA; ${ }^{6}$ Division of \\ Hematologic Malignancies and Cellular \\ Therapy, Duke University, Durham, \\ NC, USA
}

Purpose: Treatment for chronic lymphocytic leukemia (CLL) has changed dramatically with the approval of novel agents. Information regarding how patients and oncologists make trade-offs across attributes of novel therapies is limited. The purpose of this study was to understand how variations in attributes impact treatment choice among patients and oncologists.

Patients and Methods: In this study, 371 participants (patients [n=220] and oncologists $[\mathrm{n}=151]$ ) completed an online discrete choice experiment (DCE) to quantify preferences for first-line (1L) CLL treatment with novel agents; participants chose between hypothetical treatment profiles consisting of eight attributes with varying levels taken from published literature. Hierarchical Bayesian models were used to estimate attribute level preference weights. The weights were used to compute relative importance, a measure of how influential an attribute is to treatment choice.

Results: Increasing 2-year progression-free survival (PFS) from $75 \%$ to $95 \%$ had the greatest impact on preferences in 1L CLL treatment, accounting for $40 \%$ and $30 \%$ of the variation in preferences among patients and oncologists, respectively. Risk differences in atrial fibrillation (AF), infection, and discontinuation due to adverse events (AEs) were also important to patients and oncologists. Among both groups, risk differences in tumor lysis syndrome (TLS) and bleeding were least influential in treatment choice. Oncologists required 2-4 times higher increases in 2-year PFS than patients to accept increased risks of AF, discontinuation due to AEs, bleeding, TLS, and arthralgia/myalgia.

Conclusion: Patient-oncologist communication may be improved by a more focused discussion on the risks of AEs, relative to treatment outcomes, with patient goals in mind.

Keywords: leukemia, lymphocytic, chronic, B-cell, progression-free survival, tumor lysis syndrome, oncologists

\section{Introduction}

Chronic lymphocytic leukemia (CLL) accounts for approximately one-fourth of all newly diagnosed cases of leukemia in the United States (US), with most patients treated in community practice settings. A projected 21,040 patients will receive a CLL diagnosis in the US in 2020, with 4060 deaths resulting from CLL. ${ }^{1}$ The prevalence of CLL is predicted to rise by approximately 55\% between 2011 and 2025 , reflecting the results of an aging US population, the high rates of survival, and novel treatments. ${ }^{2}$ Given these trends, the number of patients with CLL who are treated in a community setting is likely to increase over time.
Correspondence: Martine C Maculaitis Email Martine.Maculaitis@kantar.com

Patient Preference and Adherence 2021:15 99-II0 
Community-based oncologists face unique challenges to providing high-quality, evidence-based care for CLL, as they often manage patients with a variety of cancer types, rather than specializing in the treatment of CLL. Thus, those who practice in the community are tasked with staying up-to-date on rapidly changing treatment advances across multiple malignancies.

Historically, chemo-immunotherapy regimens (eg, chlorambucil in combination with an anti-CD20 monoclonal antibody) were the standard of care in CLL, particularly in the first-line $(1 \mathrm{~L})$ treatment setting. However, the advent of novel targeted agents with high efficacy and improved safety profiles has led to changes in the standard of care since 2014. Among novel therapies, Bruton's tyrosine kinase (BTK) and B-cell lymphoma-2 (BCL-2) inhibitors, namely ibrutinib, acalabrutinib, and venetoclax, are considered preferred regimens for treatment-naïve (or previously untreated) patients with CLL regardless of age, comorbidities, or del (17p)/ TP53 mutation status in the current guidelines. ${ }^{3}$

With the expansion of available treatment choices, understanding the preferences of patients in treatment selection and how they view possible trade-offs are becoming more essential in treatment decision-making. Despite the growing importance of shared decision-making, the literature has yet to address key gaps between the preferences of oncologists and patients. Evidence is lacking regarding how patients and oncologists weigh efficacy, toxicity risks, and regimens when choosing $1 \mathrm{~L}$ CLL treatment, whether these groups differ in their treatment preferences, and how knowledge of such treatment preferences could guide patient-oncologist treatment discussions in the $1 \mathrm{~L}$ treatment setting. Understanding the similarities and differences in preferences between patients and oncologists is key to supporting decision-making in the $1 \mathrm{~L}$ treatment setting and ensuring treatment with novel agents is aligned with the needs and preferences of key stakeholders. This study aimed to fill these knowledge gaps by utilizing a discrete choice experiment (DCE) to determine preferences among patients and oncologists in the US with regard to key treatment attributes, including efficacy, toxicity, and route and duration of administration associated with currently approved novel therapies for CLL.

\section{Methods}

\section{Study Design}

A DCE was used to assess the preferences of patients and oncologists regarding the attributes (eg, risk of adverse events [AEs], efficacy, etc.) associated with novel agents for CLL in the $1 \mathrm{~L}$ treatment setting. DCEs have an origin in economic theory and can be applied in the healthcare setting. ${ }^{4}$ DCE can assess respondents' willingness to accept trade-offs among hypothetical treatment profiles and provides information on key attributes that drive an individual's treatment choice.

To inform the attributes incorporated in the survey, one-on-one, in-depth qualitative interviews with patients $(\mathrm{N}=10)$ and oncologists $(\mathrm{N}=10)$ using a semi-structured interview guide were conducted via telephone from April 30 to May 16, 2019 to establish which attributes were the most important to patients and oncologists. ${ }^{5}$ The verbatim interview transcripts were analyzed using qualitative data analysis software (NVivo) to identify key themes. The findings showed that efficacy, specifically long-term remission, was perceived as most important by both oncologists and patients in treatment selection. Oncologists felt that novel CLL therapies are effective and well-tolerated, although they cited some AEs, specifically tumor lysis syndrome (TLS), atrial fibrillation (AF), bleeding, infection, diarrhea, and cytopenia to be of concern. AEs that patients perceived to be of concern included bleeding, joint pain, vomiting, diarrhea, fatigue, and high blood pressure. When asked about more serious AEs, patients most frequently reported TLS, cardiac issues, serious infections, and liver issues to be of concern. Most patients reported quality of life as a consideration when evaluating treatment options. These findings and the respective risk differences in the most concerning AEs across novel therapies informed the selection and finalization of the attributes for inclusion in this study.

The final eight attributes used in the DCE survey included 2-year progression-free survival (PFS), AF (all grades), infection (grades 3/4), TLS (all grades), bleeding (grades 3/4), arthralgia/myalgia/musculoskeletal pain (all grades), discontinuation due to AEs, and duration and route of administration (Table 1). The attribute levels were identified based on US labels of approved novel agents for CLL treatment, published clinical trials, and published real-world studies.

In each DCE choice task, the participants considered two hypothetical treatment profiles, which were shown side-by-side and varied in the levels of each attribute; they were then asked to choose the treatment that was preferable to them. Appendix 1 provides a sample DCE item from the survey. The selections made by respondents allowed for an assessment of the trade-offs they were willing to make between positive and negative 
Table I Attributes and Levels Included in the DCE

\begin{tabular}{|c|c|c|}
\hline Attributes & Description & Levels, \% \\
\hline 2-year PFS & $\begin{array}{l}\text { Percent of patients will remain } \\
\text { stable (the cancer will not } \\
\text { worsen or spread) for at least } \\
2 \text { years }\end{array}$ & $\begin{array}{l}95^{20} \\
88^{21} \\
75^{22}\end{array}$ \\
\hline $\begin{array}{l}\text { Atrial fibrillation (all } \\
\text { grades) }\end{array}$ & $\begin{array}{l}\text { Percent risk of heart rhythm } \\
\text { problems (atrial fibrillation/ } \\
\text { flutter) that may require } \\
\text { medical treatment and/or } \\
\text { hospitalization }\end{array}$ & $\begin{array}{l}5^{23} \\
12^{24} \\
20^{25}\end{array}$ \\
\hline Infection (grades 3/4) & $\begin{array}{l}\text { Percent risk of infection, such } \\
\text { as pneumonia or blood } \\
\text { infections (sepsis), requiring } \\
\text { medical intervention and/or } \\
\text { hospitalization }\end{array}$ & $\begin{array}{l}7^{10} \\
17^{21,26} \\
30^{27}\end{array}$ \\
\hline TLS (all grades) & $\begin{array}{l}\text { Percent risk of TLS, which } \\
\text { requires hospitalization }\end{array}$ & $\begin{array}{l}3^{26} \\
13^{28}\end{array}$ \\
\hline Bleeding (grades 3/4) & $\begin{array}{l}\text { Percent risk of bleeding } \\
\text { (hemorrhage) that requires } \\
\text { medical intervention and/or } \\
\text { hospitalization }\end{array}$ & $\begin{array}{l}1^{10} \\
8^{27}\end{array}$ \\
\hline $\begin{array}{l}\text { Arthralgia/myalgia/ } \\
\text { musculoskeletal pain }\end{array}$ & $\begin{array}{l}\text { Percent risk of muscle, joint, or } \\
\text { bone pain }\end{array}$ & $\begin{array}{l}11^{20} \\
25^{21} \\
36^{9}\end{array}$ \\
\hline $\begin{array}{l}\text { Discontinue due to } \\
\text { AEs }\end{array}$ & $\begin{array}{l}\text { Percent risk of a side effect } \\
\text { which results in stopping the } \\
\text { medication }\end{array}$ & $\begin{array}{l}4^{10} \\
9^{24} \\
21^{28}\end{array}$ \\
\hline $\begin{array}{l}\text { Duration \& } \\
\text { administration }\end{array}$ & \multicolumn{2}{|c|}{$\begin{array}{l}\text { IV infusion therapy once a month for } 6 \\
\text { months. Along with daily oral medication } \\
\text { taken indefinitely } \\
\text { IV infusion therapy once a month for } 6 \\
\text { months. Along with daily oral medication for } \\
12 \text { months } \\
\text { No IV infusion therapy. Daily oral medication } \\
\text { taken indefinitely }\end{array}$} \\
\hline
\end{tabular}

Notes: The attributes were the components of the DCE. Description was the text that was shown to a respondent. Levels were the values at which the attributes were shown, footnoted with the reference.

Abbreviations: AE, adverse event; DCE, discrete choice exercise; IV, intravenous; PFS, progression-free survival; TLS, tumor lysis syndrome.

aspects of the treatment profiles. The levels presented for each attribute in each DCE choice task were based on a balanced design with minimal overlap. ${ }^{6}$ This design optimizes overall efficiency in terms of level balance (each level is shown approximately an equal number of times), minimal level overlap (when the levels repeat within the same task), and orthogonality (levels may be evaluated independently of other levels). All participants saw a different combination of attribute levels across the DCE choice tasks. For oncologists, the DCE presented 1800 choice tasks divided into 200 versions of nine questions; for patients, the DCE featured 2250 choice tasks divided into 250 versions of nine questions.

In addition to the DCE choice tasks, the questionnaire collected data on sociodemographic, disease, and treatment characteristics for patients and practice characteristics for oncologists. Cognitive interviews were performed with patients $(\mathrm{N}=8)$ and oncologists $(\mathrm{N}=8)$ to pilot test the survey and ensure that the survey items and instructions were easily understood.

The study was conducted in accordance with the Good Practices for Outcomes Research issued by the International Society for Pharmacoeconomics and Outcomes Research. ${ }^{7}$ The study protocol was determined to be exempt from expedited or full ethical review by the Pearl Institutional Review Board (Indianapolis, IN) prior to starting data collection, and the study conformed to US Federal Policy for the Protection of Human Subjects. Participants provided their informed consent electronically prior to study commencement.

\section{Study Sample \\ Patients}

Patients with CLL were recruited via convenience sampling utilizing cancer support and advocacy organizations, opt-in patient databases, online patient communities that had open/public forums, and referrals from physicians. The study aimed to recruit 200 patients who had a CLL diagnosis; recruitment quotas were set to ensure a roughly even split of patients who were currently receiving/had completed $1 \mathrm{~L}$ treatment (minimum $\mathrm{n}=64,32 \%$ ), were considered relapsed/refractory (R/R; minimum $\mathrm{n}=64,32 \%$ ), and were currently untreated or considered "watch and wait" patients (WW; minimum $\mathrm{n}=72,36 \%$ ) to allow sufficient statistical power to detect medium- to large-effect size mean differences (ie, Cohen's $d \geq 0.50$ ) between patient treatment subgroups. Patients were required to meet the following criteria to be eligible for the quantitative study: (1) age $\geq 18$ years, (2) a CLL diagnosis by a healthcare practitioner, (3) willingness and ability to provide informed consent, (4) English and computer literate with access to a computer, and (5) no employment with a market research, advertising, or pharmaceutical company. 


\section{Oncologists}

Oncologists were recruited from Lightspeed Research's All Global physician panel. The study sought to recruit at least 150 oncologists. Because most patients with CLL are treated in a community setting, quotas were set to ensure approximately 30\% (minimum $\mathrm{n}=45$ ) and $70 \%$ (minimum $\mathrm{n}=105$ ) of the sample were in practice in academic and community settings, respectively. Oncologists were required to meet all of the following criteria to be eligible for the quantitative study: (1) practicing between 5 and 30 years, (2) having board certification as an oncologist/hematologist, (3) currently managing $\geq 20$ patients with CLL using systemic treatments, (4) prescribing novel agents for CLL treatment (including BTK inhibitors and/or BCL-2 inhibitors), and (5) spending at least $50 \%$ of practice time in direct patient care.

\section{Statistical Analysis}

Descriptive statistics (means and standard deviations [SDs] for continuous variables and frequencies and percentages for categorical variables) were calculated for all study measures on the total samples of oncologists and patients.

The underlying choice-probability model in the hierarchical Bayes (HB) model was conditional logit, using effects coding for the attribute levels. ${ }^{8}$ The results yield the joint posterior distribution of preference weights over the entire sample, including the mean and SD for each attribute level. The preference weights measure relative preference, which means that only changes between attribute-level estimates and the relative size of those changes across attributes had meaningful interpretations. This enabled us to evaluate the magnitude of the trade-offs that oncologists and patients were willing to make among the attribute levels.

Attribute relative importance (RI) estimates demonstrated how the difference between the minimum and maximum level of each attribute contributed to the total utility of a treatment. These were calculated at the respondent level by dividing the range of each attribute (the preference weight of the most favorable level minus the preference weight of the least favorable level) by the sum of the ranges of all attributes and multiplying by 100 to convert to a percentage value. The resulting estimates reflect the importance of each attribute, relative to the others included in the DCE.

One-way analysis of variance tests (ANOVAs) or independent-samples t-tests (depending on the number of levels of the grouping variable) were used to evaluate whether preferences differed between oncologists and patients, as well as by subgroup. For oncologists, subgroups based on practice setting, years in practice, and patient volume were compared, while for patients, subgroups based on treatment status (WW vs $1 \mathrm{~L}$ vs $\mathrm{R} / \mathrm{R}$ ), age ( $<65$ years old vs $\geq 65$ years old), and community type (metropolitan/urban vs suburban vs small city/rural) were compared.

Prior to final analysis, consistency of responses across the attribute-level ratings was assessed on a scale from 1 (very bad) to 5 (very good) to flag those participants whose response patterns suggested a lack of attention, which may have resulted in biased estimates. In addition, we flagged respondents who completed the survey in less than half the median overall completion time for further evaluation. HB models were run with and without the flagged respondent data. If the findings were substantially different, the exclusion of data from these respondents from the final analysis was considered.

\section{Data Availability Statement}

The data that support the findings of this study are available for noncommercial use from the corresponding author upon reasonable request.

\section{Results}

Of the 220 patients who completed the survey, 12 had 2 or more illogical responses on the very bad/very good rating scales, 8 completed the survey in less than $50 \%$ of the median time, and 3 did both. We estimated the HB model with and without these 17 respondents. Their inclusion did not affect the HB estimates, so we decided to include their data. Of the 167 oncologists who completed the survey, 16 had 2 or more illogical responses on the very bad/very good rating scales, 19 completed the survey in less than $50 \%$ of the median time, and 7 did both. We checked the HB model estimates with and without the data from the 16 oncologists who had 2 or more illogical responses on the very bad/very good rating scales, and the preference weights estimates were impacted; thus, we decided to exclude their data from the further analysis.

\section{Sample Characteristics}

A total of 151 oncologists completed the DCE and were included in the analysis. As per recruitment quotas, most oncologists practiced in a community setting $(n=108$, $71.5 \%$ ). Most reported hematology/oncology as their 
primary medical specialty $(\mathrm{n}=117,77.5 \%)$. Oncologists reported a mean of $16.3( \pm 7.0)$ years in practice. On average, oncologists managed $65.2( \pm 64.1)$ patients with CLL in the past 12 months of which $16.6( \pm 23.2)$ were WW, $26.6( \pm 29.3)$ were $1 \mathrm{~L}$ treatment, and $22.1( \pm 21.7)$ were $\mathrm{R} / \mathrm{R}$.

A total of 220 patients completed the DCE and were included in the analysis. Patients had a mean age of 56.4 $( \pm 10.5)$ years, and three-quarters $(n=163,74.1 \%)$ selfidentified as Caucasian. On average, patients had received a CLL diagnosis for $2.0( \pm 3.1)$ years at the time of study completion. As per recruitment quotas, the majority $(\mathrm{n}=150,68.2 \%)$ had received CLL treatment $(1 \mathrm{~L}: \mathrm{n}=80$, $36.4 \% ; \mathrm{R} / \mathrm{R}: \mathrm{n}=70,31.8 \%$ ), with just under one-third $(\mathrm{n}=70,31.8 \%)$ being WW (Table 2).

\section{Oncologist and Patient Preferences}

Attribute level preference weights are shown in Figure 1. For oncologists, a change in 2-year PFS from $75 \%$ to $95 \%$ had the highest difference in preference weights (6.36). A change in the risks of AF (3.60) and treatment discontinuation due to AEs (3.18) had the next highest difference in preference weights. For patients, the same change in 2-year PFS also had the highest difference in preference weights (8.27). A change in 2-year PFS was nearly 4 times more influential to treatment preferences than changes in the risks of infection (8.27 vs 2.34) or AF (8.27 vs 2.04), the next two highest preference weights for patients.

Attribute relative importance was calculated to estimate the unique contribution to treatment choice made by each attribute across the range of preference weights. Oncologists and patients rated increasing the chance of 2-year PFS from $75 \%$ to $95 \%$ as significantly more important than improvements in any of the other attributes included in the DCE. Specifically, increasing PFS from $75 \%$ to $95 \%$ accounted for $29.5 \%$ and $40.4 \%$ of the variation in preferences among oncologists and patients, respectively. Among oncologists, decreasing the risk of AF from $20 \%$ to $5 \%$ accounted for $17.4 \%$ of the variation followed by decreasing the risk of treatment discontinuation due to AEs from $21 \%$ to $4 \%(\mathrm{RI}=16.4 \%)$ were the next most important attributes driving treatment choice. Least important were decreasing the risks of TLS (RI=4.5\%) and bleeding ( $\mathrm{RI}=5.3 \%)$ Among patients, decreasing the risks of infection from $30 \%$ to $7 \%(\mathrm{RI}=12.6 \%)$ and $\mathrm{AF}$ from $20 \%$ to $5 \%(\mathrm{RI}=11.0 \%)$ were the next most important attributes driving treatment preferences. Least important were reducing the risks of bleeding from $8 \%$ to $1 \%$
Table 2 Patient Sample Characteristics

\begin{tabular}{|c|c|c|c|}
\hline \multicolumn{2}{|l|}{ Variables } & \multirow{2}{*}{$\begin{array}{l}\mathbf{N} \\
25 \\
195\end{array}$} & \multirow{2}{*}{$\begin{array}{l}\% \\
11.36 \\
88.64\end{array}$} \\
\hline Education & $\begin{array}{l}\text { Some high school/high } \\
\text { school graduate } \\
\text { Associate degree/some } \\
\text { college or higher }\end{array}$ & & \\
\hline Marital status & $\begin{array}{l}\text { Single/separated/ } \\
\text { divorced/widowed } \\
\text { Married/committed } \\
\text { relationship }\end{array}$ & $\begin{array}{l}87 \\
133\end{array}$ & $\begin{array}{l}39.55 \\
60.45\end{array}$ \\
\hline $\begin{array}{l}\text { Employment status: } \\
\text { current }\end{array}$ & $\begin{array}{l}\text { Not employed/retired } \\
\text { Employed (full-time/part- } \\
\text { time) } \\
\text { Employed (temporary } \\
\text { leave) } \\
\text { Other }\end{array}$ & $\begin{array}{l}68 \\
106 \\
43 \\
3\end{array}$ & $\begin{array}{l}30.91 \\
48.18 \\
19.55 \\
1.36\end{array}$ \\
\hline $\begin{array}{l}\text { Employment status: } \\
\text { prior to diagnosis }\end{array}$ & $\begin{array}{l}\text { Not employed/retired } \\
\text { Employed (full-time/part- } \\
\text { time) } \\
\text { Employed (temporary } \\
\text { leave) } \\
\text { Other }\end{array}$ & $\begin{array}{l}27 \\
185 \\
3 \\
5\end{array}$ & $\begin{array}{l}12.28 \\
84.09 \\
1.36 \\
2.27\end{array}$ \\
\hline Household income & $\begin{array}{l}<\$ 50,000 \\
\geq \$ 50,000 \\
\text { Prefer not to answer }\end{array}$ & $\begin{array}{l}26 \\
182 \\
12\end{array}$ & $\begin{array}{l}6.82 \\
87.73 \\
5.45\end{array}$ \\
\hline Race/ethnicity & $\begin{array}{l}\text { African-American/black } \\
\text { Asian } \\
\text { American Indian } \\
\text { Hispanic } \\
\text { White } \\
\text { Other race/ethnicity } \\
\text { Prefer not to answer }\end{array}$ & $\begin{array}{l}33 \\
16 \\
4 \\
27 \\
163 \\
5 \\
3\end{array}$ & $\begin{array}{l}15.00 \\
7.27 \\
1.82 \\
12.27 \\
74.09 \\
2.27 \\
1.36\end{array}$ \\
\hline Community type & $\begin{array}{l}\text { Urban/metropolitan area } \\
\text { Suburb } \\
\text { Rural/small city }\end{array}$ & $\begin{array}{l}79 \\
80 \\
61\end{array}$ & $\begin{array}{l}35.91 \\
36.36 \\
27.73\end{array}$ \\
\hline Treatment status & $\begin{array}{l}\mathrm{IL} \\
\mathrm{R} / \mathrm{R} \\
\mathrm{WW}\end{array}$ & $\begin{array}{l}80 \\
70 \\
70\end{array}$ & $\begin{array}{l}36.36 \\
31.82 \\
31.82\end{array}$ \\
\hline $\begin{array}{l}\text { Age, mean } \pm \text { SD } \\
\text { Disease duration i }\end{array}$ & nean $\pm S D$ & $\begin{array}{l}56.44 \\
2.00\end{array}$ & $\begin{array}{l}10.47 \\
3.10\end{array}$ \\
\hline
\end{tabular}

Abbreviations: IL, first-line; R/R, relapsed/refractory; SD, standard deviation; WW, watch and wait.

$(\mathrm{RI}=4.7 \%)$ and $\mathrm{TLS}$ from $13 \%$ to $3 \% \quad(\mathrm{RI}=5.7 \%)$ (Figure 2).

Comparing oncologists and patients, statistically significant differences in attribute RI were observed across 2-year PFS, route of administration, and risks of AF, TLS, discontinuation due to AEs, and arthralgia/myalgia (all 


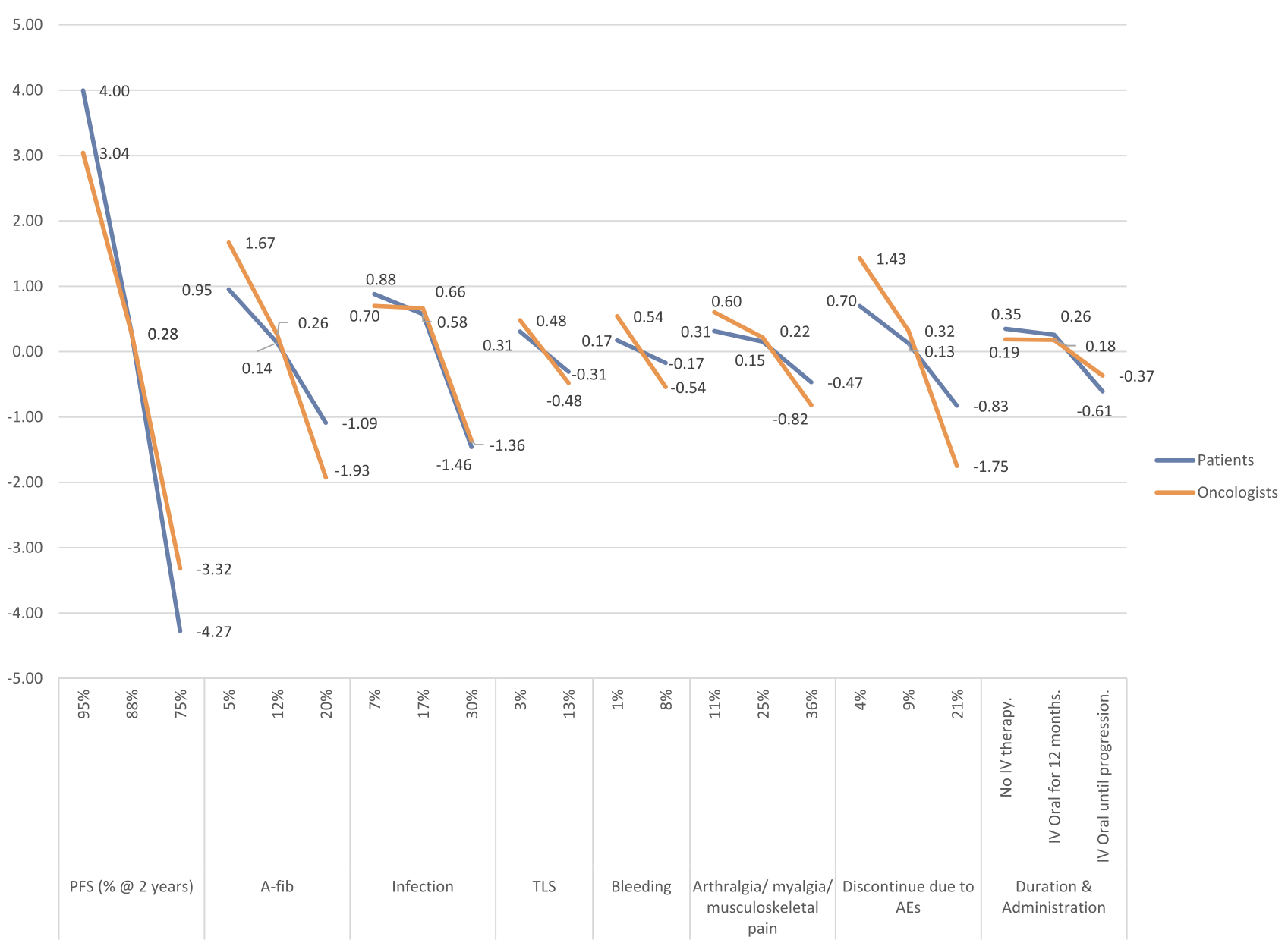

Figure I Attribute level preference weights for oncologists and patients. Preference weights measure relative preference, which means that only changes between attribute level estimates and the relative size of those changes across attributes have meaningful interpretations.

Abbreviations: AE, adverse event; IV, intravenous; PFS, progression-free survival; TLS, tumor lysis syndrome.

$P<0.01)$. The RI of risks of bleeding and infection did not vary significantly between groups (Figure 2).

\section{Subgroup Analysis}

There were no statistically meaningful differences between oncologists by practice setting, years in practice, or patient volume (Appendix 2). However, subgroup differences were observed among patients. The most clear, consistent trends were observed for age and community type. Patients $<65$ years old and those living in urban/metropolitan areas ranked 2-year PFS as more important and AErelated risks as less important than patients $\geq 65$ years old and those living in other community types, respectively (Figure 3).

\section{Trade-Offs in Relation to PFS Improvement}

Trade-offs between 2-year PFS and each of the other attributes are shown in Figure 4. The graph shows the additional percentage increase in 2-year PFS over the base level tested in the DCE (ie, 75\%) that was necessary for oncologists and patients to accept a change from the best to the worst level in each of the other attributes. Oncologists required the largest percentage increase in 2-year PFS (11.3\%) to compensate for an increased risk of $\mathrm{AF}$, whereas patients required the largest percentage increase in 2-year PFS (5.7\%) to compensate for an increased risk of infection. Oncologists required 1.1 to 4.1 times greater percentage increases in 2-year PFS than patients to compensate for increases in risks across all AE-related attributes, whereas patients required a 1.3 times greater percentage increase than oncologists in 2-year PFS in exchange for a change from receiving oral therapy to progression (no IV) to receiving oral therapy to progression plus IV therapy for 6 months. 


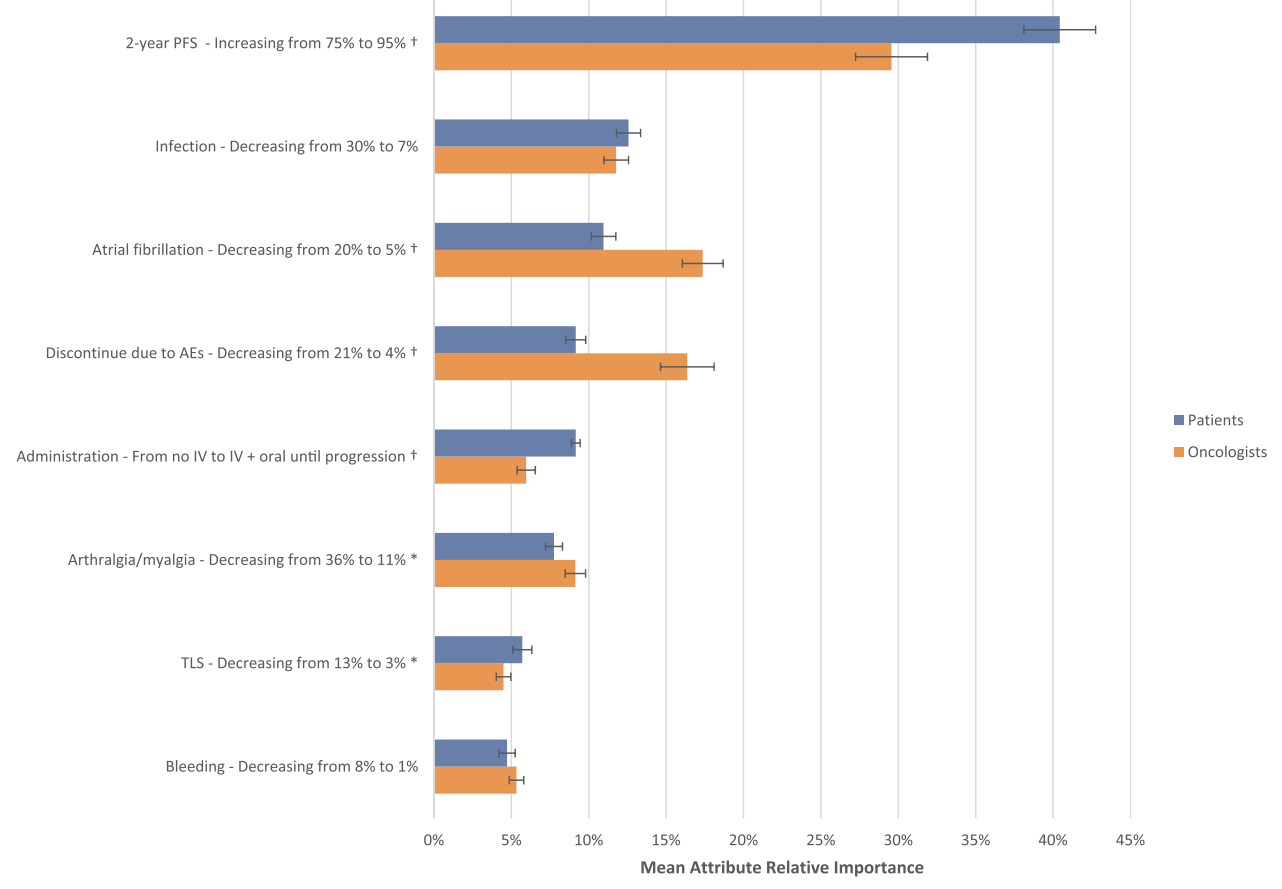

Figure 2 Relative importance of treatment attributes for oncologists and patients. Relative importance estimates are ratio scaled, so that an attribute with a relative importance of $40 \%$ is twice as important as an attribute with a relative importance of $20 \%$; $95 \%$ confidence intervals are shown in the error bars. Symbols represent a statistically significant difference between groups at $(*) P<0.0 \mathrm{I}$ and $\left(^{\dagger}\right) P<0.00 \mathrm{I}$, two-tailed.

Abbreviations: $A E$, adverse event; IV, intravenous; PFS, progression-free survival; TLS, tumor lysis syndrome.

\section{Discussion}

The current study provides unique insights into treatment preferences in the $1 \mathrm{~L}$ treatment setting among patients with CLL and oncologists in the US. The highest value was placed on improvements in 2-year PFS by both oncologists and patients, but they also valued safety, especially decreased risks of $\mathrm{AF}$, infection, and discontinuation due to AEs. Indeed, the largest increases in the percentage change of 2-year PFS would be required to compensate for an increased risk of these AEs. Notably, patients placed greater weight than oncologists on an increase in 2-year PFS when selecting a novel therapy for CLL. While both patients and oncologists were willing to make trade-offs to obtain incremental 2-year PFS benefits, oncologists required much greater improvements in efficacy than patients to offset the higher risks of AEs or treatment discontinuation due to AEs.

In the in-depth qualitative interviews conducted in the initial phase of the study, all of the attributes selected for the DCE were perceived as being important to treatment decision-making by patients and oncologists. ${ }^{5}$ Although route of administration and risks of TLS and bleeding were mentioned spontaneously as being of high importance in the qualitative interviews, these attributes were ranked lower than the others included in the DCE choice tasks. This discrepancy may potentially be because the DCE choice task forces the respondent to consider the hypothetical treatment regimen as a collective set of attributes and levels, rather than evaluating attributes in isolation of other factors that may influence treatment choice.

Major bleeding was one of the least important attributes to patients and oncologists in the current study. While this AE is associated with BTK inhibitors, ${ }^{9,10}$ results may reflect perceptions that major bleeding has a low likelihood of occurrence, which is consistent with the range of the levels (1-8\%) included for this attribute in the DCE. Oncologists perceived TLS to be the least important attribute, overall. This result may be a function of the infrequent occurrence of TLS in routine clinical practice or owing to the success of TLS risk-stratification and dose ramp-up strategies at preventing serious TLS. ${ }^{11}$

In a previous systematic review, patients with cancer preferred oral treatment over IV therapy in $85 \%$ of the studies evaluated, ${ }^{12}$ which was consistent with the indepth qualitative research we initially conducted. ${ }^{5}$ Our study found similar results with patients and oncologists preferring regimens without IV components. However, preferences for route of administration were superseded in the DCE by preferences for higher efficacy and lower 


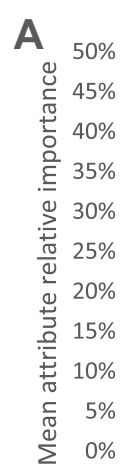

\section{B}

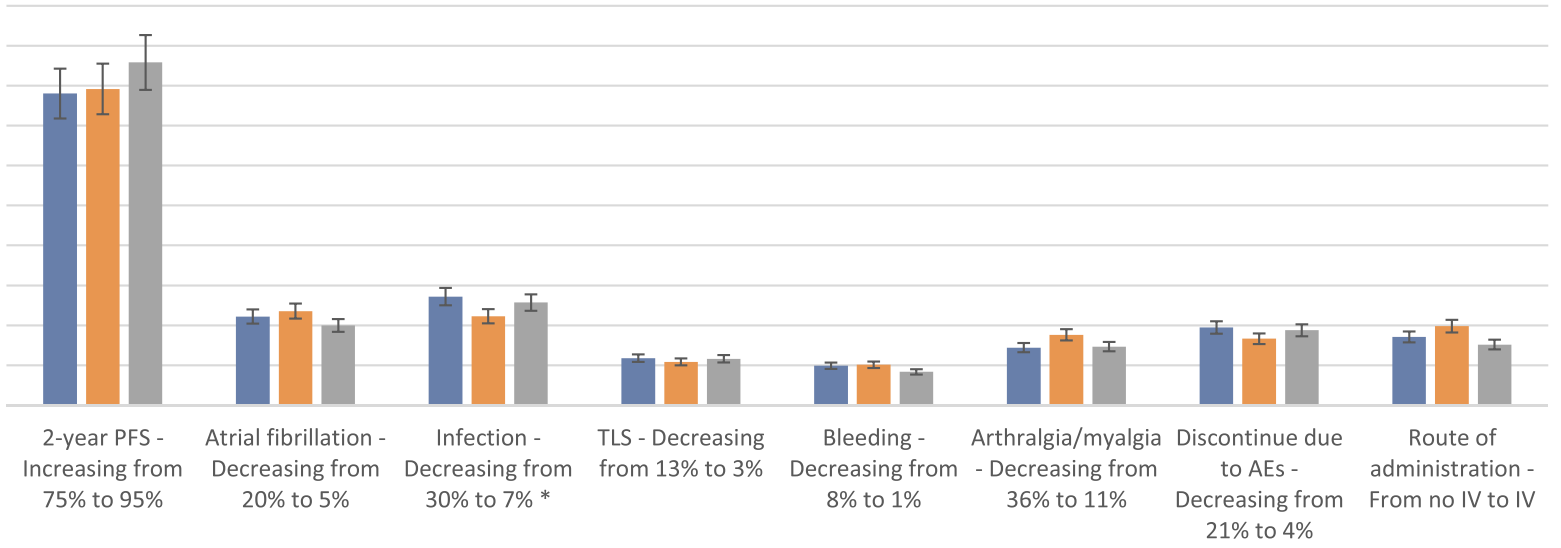

$\square \mathrm{L}=\mathrm{R} / \mathrm{R} \quad \mathrm{WW}$

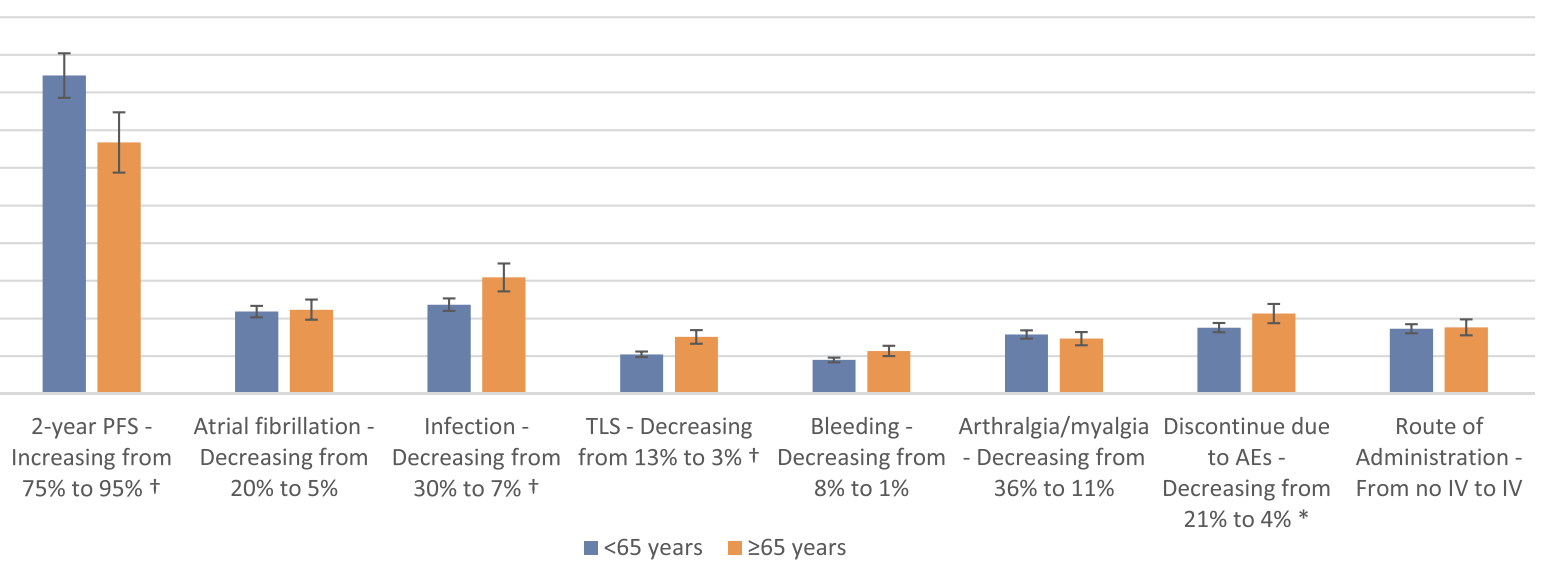

C

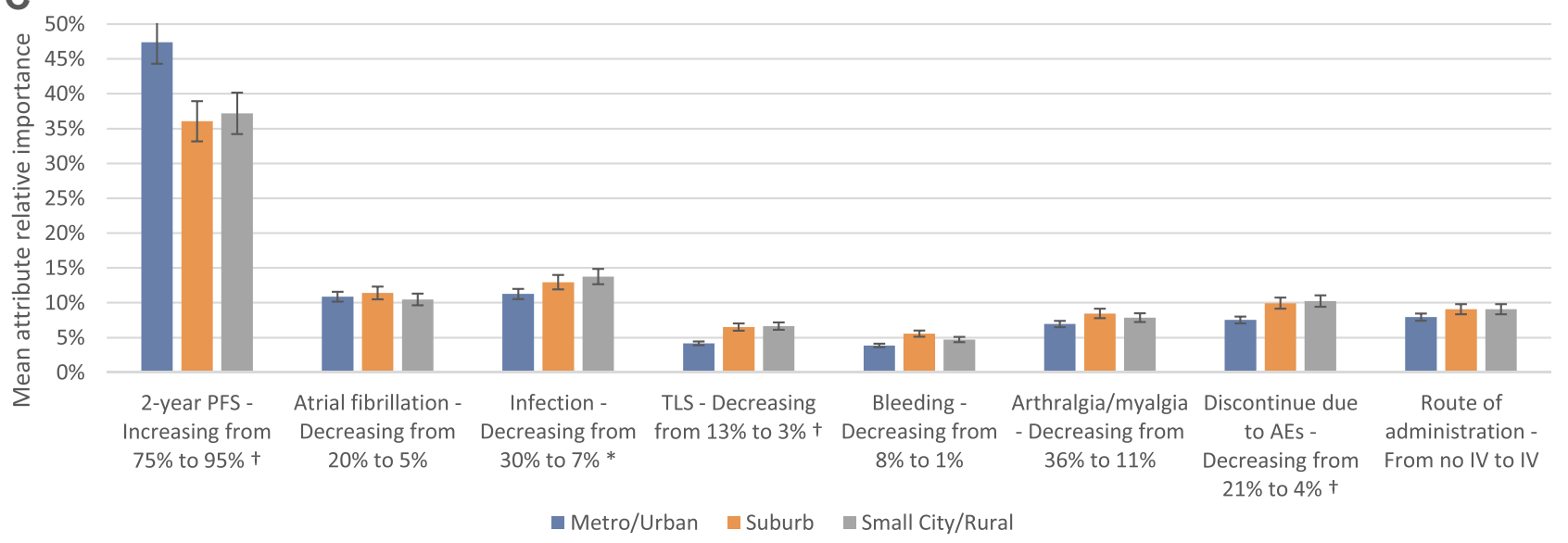

Figure 3 Relative importance of treatment attributes by patient subgroup. Relative importance estimates are ratio scaled, so that an attribute with a relative importance of $40 \%$ is twice as important as an attribute with a relative importance of $20 \%$; $95 \%$ confidence intervals are shown in the error bars. Symbols represent a statistically significant difference between groups at $\left({ }^{*}\right) P<0.05$ and $\left({ }^{\dagger}\right) P<0.0$ I, two-tailed. (A) Relative importance by patient treatment status. (B) Relative importance by patient age. (C) Relative importance by patient community type.

Abbreviations: IL, first-line; AE, adverse event; IV, intravenous; PFS, progression-free survival; R/R, relapsed/refractory; TLS, tumor lysis syndrome; WW, watch and wait.

risks of AEs. Route of administration may be more highly prioritized by patients and oncologists in light of the COVID-19 pandemic, although additional research is needed to confirm this possibility.
Subgroup analyses showed that patients $<65$ years old and those living in urban/metropolitan areas ranked 2-year PFS as more important and AE-related risks as less important than patients $\geq 65$ years old and those living in other 


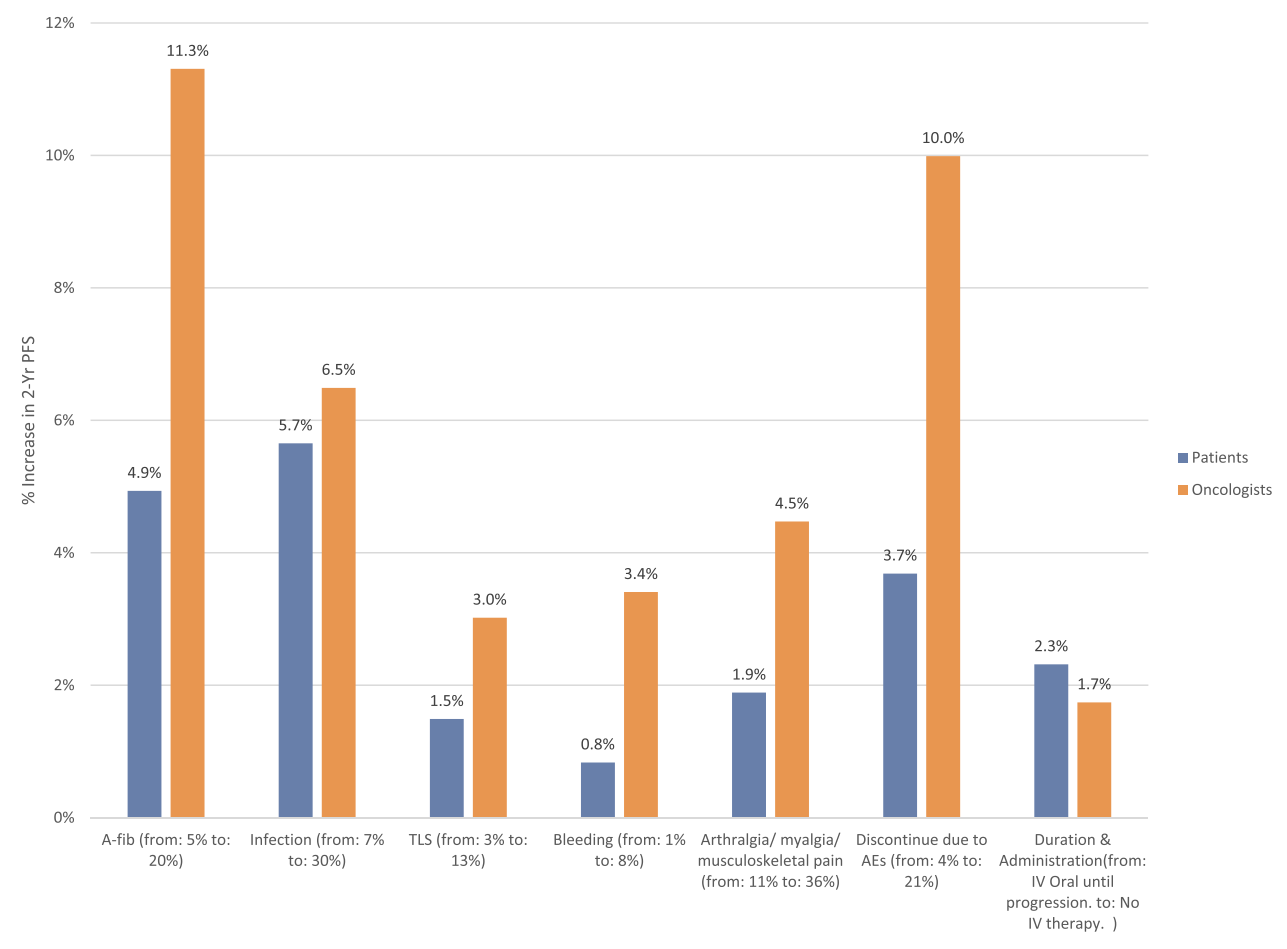

Figure 4 Trade-offs in percentage 2-year PFS required for oncologists and patients. Abbreviations: AE, adverse event; IV, intravenous; TLS, tumor lysis syndrome.

community types, respectively. Prior research has indicated that, among rural adults with cancer, greater travel times to the hospital or specialty cancer center are associated with receiving less medical care. ${ }^{13}$ Accordingly, patients residing in small cities/rural areas may put greater emphasis on avoiding AEs when selecting CLL treatment because of difficulties in accessing the healthcare resources that are more readily available in urban locales. Our results are supportive of this hypothesis, although further research is required to validate this theory for patients with CLL. Younger patients also placed more value on 2-year PFS. It is possible that younger patients are more interested in achieving greater longevity, whereas, for older patients, an additional year of life may be less valuable to them than it is to a younger patient, thus prioritizing quality of life over quantity. Given that results for both urban community type and younger age were aligned, there may be a potential confounding effect due to demographic trends in which a disproportionate share of US adults aged $\geq 65$ years reside in rural communities. ${ }^{14}$

The results of the current study are consistent with past research by Mansfield regarding the primary importance of PFS to patients, ${ }^{15}$ with the present study confirming a similar level of importance for this attribute among patients and oncologists. The present study is unique in its assessment and comparison of both patients and oncologists in the same study in a US sample. Whereas overall survival was found to be the most important factor in a previous European study of providers, patients, and the lay public, ${ }^{16}$ the current study did not assess this attribute, as it was found to be of lesser importance than PFS to CLL treatment decisions in the initial qualitative phase of this study. ${ }^{5}$ Importantly, in a study by Landfeldt and colleagues, ${ }^{16}$ route of administration was found to be more important to patients than providers, a finding that is consistent with the results of the current study and reinforces the need to consider the preferences of all stakeholders in discussions of treatment options in CLL.

The current study addressed key gaps in the limited prior literature on stakeholder preferences in CLL. Specifically, prior studies, which were published in 2016-2017, ${ }^{15-17}$ did not include attributes and levels representing a broader array of novel therapies, as only ibrutinib was approved at that time in the $1 \mathrm{~L}$ treatment setting. Instead, these earlier studies included choice tasks with chemotherapy and focused on the $R / R$ setting. In addition, the few DCE studies conducted on treatment preferences in CLL have focused on the treatment preferences of multiple European stakeholders, ${ }^{16,17}$ and the sole 
US-based study only evaluated the preferences of patients. ${ }^{15}$ As such, the perspectives of a broader array of key US stakeholders had been missing. The present study makes important contributions to the understanding of US patient and oncologist preferences in the context of the most current standards of care in $1 \mathrm{~L}$ treatment for CLL.

Prior research has shown that when treatment decisions are aligned with patient preferences, patients report greater treatment satisfaction and adherence and have better clinical outcomes. ${ }^{18}$ Furthermore, patients who take a more active role in decisions about their own healthcare have fewer emergency room visits and hospitalizations. ${ }^{19}$ As a consequence, although there was considerable alignment in oncologist and patient preferences in the current study, the areas where they diverge warrant closer attention. It is important for oncologists to consider those aspects of treatment that are most valued by their patients when making treatment recommendations, and to recognize that patients' preferences may differ from oncologists' own preferences. As we increasingly follow a shared decision-making model in clinical practice, eliciting patients' values and preferences is particularly important.

\section{Limitations}

The current study provides novel insights into the preferences of patients and oncologists; however, the following limitations should be noted. Our study used a convenience sampling technique, which may have led to a sample that is not generalizable to the broader CLL population. It is possible that younger, healthier patients or those interested in research may have been more likely to participate in the study, resulting in selection bias. Of note, the mean age of our study population was below the mean age of diagnosis for CLL. To address that limitation, subgroup analyses were conducted to evaluate the potential impact of age and other patient characteristics on preferences. Further, study variables were self-reported and could not be independently verified; self-reported data may also be subject to response bias, which can increase measurement error. Due to small sample sizes, oncologist subgroup comparisons may have been insufficiently powered to detect statistically significant differences. In addition, treatment preferences could differ for patients who have previously participated in a clinical trial, although this possibility will need to be examined in a future study with a larger sample of patients with this experience. Additionally, concerns about polypharmacy and potential interactions of novel therapies with medications for comorbid health conditions may influence treatment preferences with respect to different patient subpopulations than the ones included in the current study.

In addition to biases pertaining to study sampling, the DCE choice tasks may not reflect the same clinical, economic, or personal consequences of real-world treatment decisions. Notably, self-reported preferences may diverge from actual treatment decisions, and the DCE cannot include all possible factors that may underlie individuals' treatment preferences. For instance, costs may influence preferences, and we cannot exclude the possibility that preferences may have varied if treatment costs had been considered among the attributes presented, particularly given the high costs of novel therapies. However, treatment cost was not considered in this study, as our primary focus was on how efficacy-, safety-, and dosing-related attributes influence preferences. Furthermore, grouping together administration form (IV vs oral) and frequency/duration of therapy (treat to progression vs treat for 12 months vs treat for 24 months) into a single attribute may have masked the unique contribution of each of these factors to treatment preferences. This is an area that would benefit from further research, given the increased number of treatment options available for CLL. Although choice tasks are not the same as making an individual treatment selection for a patient, the DCE was carefully designed to include the feedback of the two stakeholder groups of interest, to mimic realistic $1 \mathrm{~L}$ treatment options for CLL, and to closely align with the clinical evidence available at the time that the study was conducted.

\section{Conclusions}

This study elucidates the importance of PFS to oncologists and patients with CLL in the era of novel targeted therapies. It adds to existing literature by quantifying the tradeoffs that these groups are willing to make to avoid potential risks of AEs and associated treatment discontinuation. Important differences between the two groups emerged, with oncologists requiring much higher increases in PFS than patients to accept increased risks of AEs and treatment discontinuation. These data suggest that patients and oncologists may perceive the risks and benefits associated with novel agents differently. Patient-oncologist communication could be enhanced through a discussion of the risks of AEs, relative to treatment outcomes, with a focus on available novel therapies. 


\section{Abbreviations}

1L, first-line; AE, adverse event; AF, atrial fibrillation; BCL2, B-cell lymphoma-2; BTK, Bruton's tyrosine kinase; CLL, chronic lymphocytic leukemia; DCE, discrete choice experiment; PFS, progression-free survival; RI, relative importance; TLS, tumor lysis syndrome; US, United States.

\section{Acknowledgments}

The study was funded by AstraZeneca. The authors acknowledge Thomas Campbell, $\mathrm{PhD}$, for his input on the study design, Kathleen Beusterien, MPH, for her input on interpretation of results, and Errol J. Philip, $\mathrm{PhD}$, for his assistance with manuscript preparation.

\section{Author Contributions}

All authors made a significant contribution to the work reported, whether that is in the conception, study design, execution, acquisition of data, analysis and interpretation, or in all these areas; took part in drafting, revising or critically reviewing the article; gave final approval of the version to be published; have agreed on the journal, which the article has been submitted; and agree to be accountable for all aspects of the work.

\section{Funding}

This study was funded by AstraZeneca. Kantar received funding from AstraZeneca to conduct and report on this study.

\section{Disclosure}

HL, KR, and SKW: Employees of AstraZeneca. MCM, EM, and OW: Employees of Kantar, which received funding from AstraZeneca to conduct this study. TWL: Personal fees for consulting or advisory boards: AbbVie, Agios, Amgen, AstraZeneca, BMS/Celgene, CareVive, DaiichiSankyo, Otsuka, Pfizer, Seattle Genetics; Royalties: UpToDate; Speakers bureaus/honoraria: AbbVie, Agios, BMS/Celgene; Grants/research contracts: American Cancer Society, AstraZeneca, CareVive, Duke University, Flatiron, Helsinn, Heron, Jazz Pharmaceuticals, NINR/NIH, Seattle Genetics. The authors report no other conflicts of interest in this work.

\section{References}

1. Key statistics for chronic lymphocytic leukemia [page on the Internet]. American Cancer Society; January 8, 2020. Available from: https:// www.cancer.org/cancer/chronic-lymphocytic-leukemia/about/keystatistics.html. Accessed June 15, 2020.
2. Chen Q, Jain N, Ayer T, et al. Economic burden of chronic lymphocytic leukemia in the era of oral targeted therapies in the United States. J Clin Oncol. 2017;35(2):166-174. doi:10.1200/JCO.2016.68. 2856

3. Hallek M. Chronic lymphocytic leukemia: 2020 update on diagnosis, risk stratification and treatment. Am J Hematol. 2019;94(11): 1266-1287. doi:10.1002/ajh.25595

4. Clark MD, Determann D, Petrou S, Moro D, de Bekker-grob EW. Discrete choice experiments in health economics: a review of the literature. Pharmacoeconomics. 2014;32(9):883-902.

5. Le H, Pendergraft T, Wahlstrom SK, et al. Understanding clinician and patient preferences about novel agents in chronic lymphocytic leukemia [abstract]. Blood. 2019;134(Supp11):4730. doi:10.1182/ blood-2019-129606

6. Johnson FR, Lancsar E, Marshall D, et al. Constructing experimental designs for discrete-choice experiments: report of the ISPOR conjoint analysis experimental design good research practices task force. Value Health. 2013;16(1):3-13. doi:10.1016/j.jval.2012.08.2223

7. Bridges JF, Hauber AB, Marshall D, et al. Conjoint analysis applications in health - a checklist: a report of the ISPOR good research practices for conjoint analysis task force. Value Health. 2011;14 (4):403-413. doi:10.1016/j.jval.2010.11.013

8. Hauber AB, González JM, Groothuis-Oudshoorn CGM. Statistical methods for the analysis of discrete choice experiments: a report of the ISPOR conjoint analysis good research practices task force. Value Health. 2016;19(4):300-315. doi:10.1016/j.jval.2016.04.004

9. Burger JA, Tedeschi A, Barr PM, et al. Ibrutinib as initial therapy for patients with chronic lymphocytic leukemia. New Eng J Med. 2015;373:2425-2437. doi:10.1056/NEJMoa1509388

10. Byrd JC, Brown JR, O'Brien S, et al. Ibrutinib versus ofatumumab in previously treated chronic lymphoid leukemia. New Eng $J$ Med. 2014;371:213-223. doi:10.1056/NEJMoa1400376

11. Roeker LE, Fox CP, Eyre TA, et al. Tumor lysis, adverse events, and dose adjustments in 297 venetoclax-treated CLL patients in routine clinical practice. Clin Cancer Res. 2019;25(14):4264-4270. doi:10.11 58/1078-0432.CCR-19-0361

12. Eek D, Krohe M, Mazar I, et al. Patient-reported preferences for oral versus intravenous administration for the treatment of cancer: a review of the literature. Patient Prefer Adherence. 2016;10: 1609-1621. doi:10.2147/PPA.S106629

13. Baird G, Flynn R, Baxter G, Donnelly M, Lawrrence J. Travel time and cancer care: an example of the inverse care law? Rural Remote Health. 2008;8:1003.

14. Demographic and economic trends in urban, suburban and rural communities [internet]. Pew Research Center; 2018. Available from: https://www.pewsocialtrends.org/2018/05/22/demographic-and -economic-trends-in-urban-suburban-and-rural-communities/. Accessed April 10, 2020.

15. Mansfield C, Masaquel A, Sutphin J, et al. Patients' priorities in selecting chronic lymphocytic leukemia treatments. Blood $A d v$. 2017;1(24):2176-2185. doi:10.1182/bloodadvances.2017007294

16. Landfeldt E, Eriksson J, Ireland S, et al. Patient, physician, and general population preferences for treatment characteristics in relapsed or refractory chronic lymphocytic leukemia: a conjoint analysis. Leuk Res. 2016;40:17-23. doi:10.1016/j.leukres.2015.11.006

17. Boqué C, Abad M, Moreno C, et al. Healthcare professionals' preferences for the treatment selection of chronic lymphocytic leukemia (CLL): the Prelic Study [abstract]. Value Health. 2017;20:A455. doi:10.1016/j.jval.2017.08.319.

18. Lindhiem O, Bennett CB, Trentacosta CJ, McLear C. Client preferences affect treatment satisfaction, completion, and clinical outcome: a meta-analysis. Clin Psychol Rev. 2014;34(6):506-517. doi:10.1016/ j.cpr.2014.06.002

19. Greene J, Hibbard JH, Sacks R, Overton V, Parrotta CD. When patient activation levels change, health outcomes and costs change, too. Health Aff. 2015;34(3):431-437. doi:10.1377/hlthaff.2014.0452 
20. Sharman JP, Banerji V, Fogliatto LM, et al. ELEVATE TN: Phase 3 study of acalabrutinib combined with obinutuzumab $(\mathrm{O})$ or alone vs $\mathrm{O}$ plus chlorambucil $(\mathrm{Clb})$ in patients (Pts) with treatment-naive chronic lymphocytic leukemia (CLL) [abstract]. Blood. 2019;134 (Suppl1):31. doi:10.1182/blood-2019-128404

21. Fischer K, Al-Sawaf O, Fink A-M, et al. Venetoclax and obinutuzu$\mathrm{mab}$ in chronic lymphocytic leukemia. Blood. 2017;129 (19):2702-2705. doi:10.1182/blood-2017-01-761973

22. Farooqui MZ, Valdez J, Martyr S, et al. Ibrutinib for previously untreated and relapsed or refractory chronic lymphocytic leukaemia with TP53 aberrations: a Phase 2, single-arm trial. Lancet Oncol. 2015;16(2):169-176. doi:10.1016/S1470-2045(14)71 182-9

23. Ghia P, Pluta A, Wach M, et al. Acalabrutinib vs rituximab plus idelalisib (IdR) or bendamustine (BR) by investigator choice in relapsed/refractory (RR) chronic lymphocytic leukemia: phase 3 ASCEND study [abstract]. Hematol Oncol. 2019;37:86-87. doi:10.1002/hon.54 2629.

24. Moreno C, Greil R, Demirkan F, et al. Ibrutinib plus obinutuzumab versus chlorambucil plus obinutuzumab in first-line treatment of chronic lymphocytic leukaemia (iLLUMINATE): a multicentre, randomised, open-label, phase 3 trial. Lancet Oncol. 2019;20(1):43-56. doi:10.1016/S1470-2045(18)30788-5
25. Tedeschi A Clinical impact of new data in B-cell malignancies from EHA and ICML 2019. Paper presented at: Annual Congress of the European Haematolgical Association; June 13-16, 2019; Amsterdam, Netherlands.

26. Seymour JF, Kipps TJ, Eichhorst B, et al. Venetoclax-rituximab in relapsed or refractory chronic lymphocytic leukemia. $N$ Engl J Med. 2018;378(12):1107-1120. doi:10.1056/NEJMoa1713976

27. O'Brien S, Jones JA, Coutre SE, et al. Ibrutinib for patients with relapsed or refractory chronic lymphocytic leukaemia with $17 \mathrm{p}$ deletion (RESONATE-17): a phase 2, open-label, multicentre study. Lancet Oncol. 2016;17(10):1409-1418. doi:10.1016/S1470-2045(16) 30212-1

28. Mato AR, Thompson M, Allan JN, et al. Real-world outcomes and management strategies for venetoclax-treated chronic lymphocytic leukemia patients in the United States. Haematologica. 2018;103 (9):1511-1517. doi:10.3324/haematol.2018.193615

29. IMBRUVICA ${ }^{\circledR}$ (Ibrutinib) [Package Insert]. Sunnyvale, CA: Pharmacyclics LLC; 2020.

30. GAZYVA ${ }^{\circledR}$ (Obinutuzumab) [Package Insert]. South San Francisco, CA: Genentech, Inc; 2017.

31. VENCLEXTA ${ }^{\circledR}$ (Venetoclax) [Package Insert]. North Chicago, IL: AbbVie Inc; 2016.
Patient Preference and Adherence

\section{Publish your work in this journal}

Patient Preference and Adherence is an international, peer-reviewed, open access journal that focusing on the growing importance of patient preference and adherence throughout the therapeutic continuum. Patient satisfaction, acceptability, quality of life, compliance, persistence and their role in developing new therapeutic modalities and compounds to optimize clinical outcomes for existing disease

\section{Dovepress}

states are major areas of interest for the journal. This journal has been accepted for indexing on PubMed Central. The manuscript management system is completely online and includes a very quick and fair peer-review system, which is all easy to use. Visit http:// www.dovepress.com/testimonials.php to read real quotes from published authors. 